\title{
HESS observations and VLT spectroscopy of PG 1553+113
}

F. Aharonian ${ }^{1,13}$, A. G. Akhperjanian ${ }^{2}$, U. Barres de Almeida ${ }^{8, \star}$, A. R. Bazer-Bachi ${ }^{3}$, B. Behera ${ }^{14}$, M. Beilicke ${ }^{4}$, W. Benbow ${ }^{1}$, K. Bernlöhr ${ }^{1,5}$, C. Boisson ${ }^{6}$, O. Bolz ${ }^{1}$, V. Borrel ${ }^{3}$, I. Braun ${ }^{1}$, E. Brion ${ }^{7}$, A. M. Brown ${ }^{8}$, R. Bühler ${ }^{1}$, T. Bulik ${ }^{24}$, I. Büsching ${ }^{9}$, T. Boutelier ${ }^{17}$, S. Carrigan ${ }^{1}$, P. M. Chadwick ${ }^{8}$, L.-M. Chounet ${ }^{10}$, A. C. Clapson ${ }^{1}$, G. Coignet $^{11}$, R. Cornils ${ }^{4}$, L. Costamante ${ }^{1,28}$, M. Dalton ${ }^{5}$, B. Degrange ${ }^{10}$, H. J. Dickinson ${ }^{8}$, A. Djannati-Atai ${ }^{12}$, W. Domainko ${ }^{1}$, L. O'C. Drury ${ }^{13}$, F. Dubois ${ }^{11}$, G. Dubus ${ }^{17}$, J. Dyks ${ }^{24}$, K. Egberts ${ }^{1}$, D. Emmanoulopoulos ${ }^{14}$, P. Espigat ${ }^{12}$, C. Farnier $^{15}$, F. Feinstein ${ }^{15}$, A. Fiasson ${ }^{15}$, A. Förster ${ }^{1}$, G. Fontaine ${ }^{10}$, Seb. Funk ${ }^{5}$, M. Füßling ${ }^{5}$, Y. A. Gallant ${ }^{15}$, B. Giebels ${ }^{10}$, J. F. Glicenstein ${ }^{7}$, B. Glück ${ }^{16}$, P. Goret ${ }^{7}$, C. Hadjichristidis ${ }^{8}$, D. Hauser ${ }^{1}$, M. Hauser ${ }^{14}$, G. Heinzelmann ${ }^{4}$, G. Henri ${ }^{17}$, G. Hermann ${ }^{1}$, J. A. Hinton ${ }^{25}$, A. Hoffmann ${ }^{18}$, W. Hofmann ${ }^{1}$, M. Holleran ${ }^{9}$, S. Hoppe ${ }^{1}$, D. Horns ${ }^{18}$, A. Jacholkowska ${ }^{15}$, O. C. de Jager ${ }^{9}$, I. Jung ${ }^{16}$, K. Katarzyński ${ }^{27}$, E. Kendziorra ${ }^{18}$, M. Kerschhaggl ${ }^{5}$, B. Khélifi ${ }^{10}$, D. Keogh ${ }^{8}$, Nu. Komin ${ }^{15}$, K. Kosack ${ }^{1}$, G. Lamanna ${ }^{11}$, I. J. Latham ${ }^{8}$, A. Lemière ${ }^{12}$, M. Lemoine-Goumard ${ }^{10}$, J.-P. Lenain ${ }^{6}$, T. Lohse ${ }^{5}$, J. M. Martin ${ }^{6}$, O. Martineau-Huynh ${ }^{19}$, A. Marcowith ${ }^{15}$, C. Masterson ${ }^{13}$, D. Maurin ${ }^{19}$, G. Maurin ${ }^{12}$, T. J. L. McComb ${ }^{8}$, R. Moderski ${ }^{24}$, E. Moulin ${ }^{7}$, M. de Naurois ${ }^{19}$, D. Nedbal ${ }^{20}$, S. J. Nolan ${ }^{8}$, S. Ohm ${ }^{1}$, J.-P. Olive ${ }^{3}$, E. de Oña Wilhelmi ${ }^{12}$, K. J. Orford ${ }^{8}$, J. L. Osborne ${ }^{8}$, M. Ostrowski ${ }^{23}$, M. Panter ${ }^{1}$, G. Pedaletti ${ }^{14}$, G. Pelletier ${ }^{17}$, P.-O. Petrucci ${ }^{17}$, S. Pita ${ }^{12}$, G. Pühlhofer ${ }^{14}$, M. Punch ${ }^{12}$, S. Ranchon ${ }^{11}$, B. C. Raubenheimer ${ }^{9}$, M. Raue ${ }^{4}$, S. M. Rayner ${ }^{8}$, M. Renaud ${ }^{1}$, J. Ripken ${ }^{4}$, L. Rob ${ }^{20}$, L. Rolland ${ }^{7}$, S. Rosier-Lees ${ }^{11}$, G. Rowell ${ }^{26}$, B. Rudak ${ }^{24}$, J. Ruppel ${ }^{21}$, V. Sahakian ${ }^{2}$, A. Santangelo ${ }^{18}$,

R. Schlickeiser ${ }^{21}$, F. Schöck ${ }^{16}$, R. Schröder ${ }^{21}$, U. Schwanke ${ }^{5}$, S. Schwarzburg ${ }^{18}$, S. Schwemmer ${ }^{14}$, A. Shalchi ${ }^{21}$, H. Sol ${ }^{6}$, D. Spangler ${ }^{8}$, Ł. Stawarz ${ }^{23}$, R. Steenkamp ${ }^{22}$, C. Stegmann ${ }^{16}$, G. Superina ${ }^{10}$, P. H. Tam ${ }^{14}$, J.-P. Tavernet ${ }^{19}$, R. Terrier ${ }^{12}$, C. van Eldik $^{1}$, G. Vasileiadis ${ }^{15}$, C. Venter ${ }^{9}$, J. P. Vialle ${ }^{11}$, P. Vincent ${ }^{19}$, M. Vivier ${ }^{7}$, H. J. Völk ${ }^{1}$, F. Volpe ${ }^{10}$, S. J. Wagner ${ }^{14}$, M. Ward ${ }^{8}$, A. A. Zdziarski ${ }^{24}$, and A. Zech ${ }^{6}$

(Affiliations can be found after the references)

Received 3 September 2007 / Accepted 25 October 2007

\section{ABSTRACT}

Aims. The properties of the very high energy (VHE; $E>100 \mathrm{GeV}$ ) $\gamma$-ray emission from the high-frequency peaked BL Lac PG $1553+113$ are investigated. An attempt is made to measure the currently unknown redshift of this object.

Methods. VHE Observations of PG 1553+113 were made with the High Energy Stereoscopic System (HESS) in 2005 and $2006 . H+$ $K(1.45-2.45 \mu \mathrm{m})$ spectroscopy of PG 1553+113 was performed in March 2006 with SINFONI, an integral field spectrometer of the ESO Very Large Telescope (VLT) in Chile.

Results. A VHE signal, $\sim 10$ standard deviations, is detected by HESS during the 2 years of observations ( $24.8 \mathrm{~h}$ live time). The integral flux above $300 \mathrm{GeV}$ is $\left(4.6 \pm 0.6_{\text {stat }} \pm 0.9_{\text {syst }}\right) \times 10^{-12} \mathrm{~cm}^{-2} \mathrm{~s}^{-1}$, corresponding to $\sim 3.4 \%$ of the flux from the Crab Nebula above the same threshold. The time-averaged energy spectrum is measured from $225 \mathrm{GeV}$ to $\sim 1.3 \mathrm{TeV}$, and is characterized by a very soft power law (photon index of $\Gamma=4.5 \pm 0.3_{\text {stat }} \pm 0.1_{\text {syst }}$ ). No evidence for any flux or spectral variations is found on any sampled time scale within the VHE data. The redshift of PG 1553+113 could not be determined. Indeed, even though the measured SINFONI spectrum is the most sensitive ever reported for this object at near infrared wavelengths, and the sensitivity is comparable to the best spectroscopy at other wavelengths, no absorption or emission lines were found in the $\mathrm{H}+\mathrm{K}$ spectrum presented here.

Key words. galaxies: active - galaxies: BL Lacertae objects: individual: PG 1553+113 - gamma rays: observations

\section{Introduction}

Evidence for VHE $(>100 \mathrm{GeV}) \gamma$-ray emission from the active galactic nucleus (AGN) PG $1553+113$ was first reported by the HESS collaboration (Aharonian et al. 2006a) based on observations made in 2005. This evidence was later confirmed (Albert et al. 2007) with MAGIC observations in 2005 and 2006. Similar to essentially all AGN detected at VHE energies, PG 1553+113 is classified as a high-frequency peaked BL Lac (Giommi et al. 1995) and is therefore believed to possess the double-humped broad-band spectral energy distribution (SED) typical of blazars. The low-energy (i.e. from the radio to the X-ray regime)

* Supported by CAPES Foundation, Ministry of Education of Brazil. portion of the SED of PG $1553+113$ is well-studied, including several simultaneous multi-wavelength observation campaigns (see, e.g., Osterman et al. 2006). However, the only data in the high-energy hump are from HESS and MAGIC. The measured VHE spectra are unusually soft (photon index $\Gamma=4.0 \pm 0.6$ and $\Gamma=4.2 \pm 0.3$, respectively) but the errors are large, clearly requiring improved measurements before detailed interpretation of the complete SED is possible.

Further complicating any SED interpretation is the absorption of VHE photons (Gould \& Schréder 1967; Hauser \& Dwek 2001) by pair-production on the Extragalactic Background Light (EBL). This absorption, which is energy dependent and increases strongly with redshift, distorts the VHE energy 
spectra observed from distant objects. For a given redshift and a given EBL model, the effects of the latter on the observed spectrum can be reasonably accounted for during SED modeling. Unfortunately, the redshift of PG $1553+113$ is currently unknown ${ }^{1}$. To date no emission or absorption lines have been measured from PG $1553+113$ despite more than ten observation campaigns with optical instruments, including EMMI at the NTT and FORS2 with the 8-m VLT telescopes (Carangelo et al. 2003; Sbarufatti et al. 2006). Lower limits of $z>0.09$ (Sbarufatti et al. 2006) and $z>0.3$ (Carangelo et al. 2003) were determined from the lack of detected absorption/emission lines, implying that the effect of the EBL is large in the observed VHE data. The absence of absorption and emission lines suggests that the non-thermal component of the emission from PG $1553+113$ is largely dominant over that of the host galaxy. This is consistent with the fact that, although some hints for a host galaxy have been suspected, no clear detection has yet been found, even in Hubble Space Telescope (HST) images of PG $1553+113$ taken during the HST survey of 110 BL Lac objects (Scarpa et al. 2000). Interestingly, approximately $80 \%$ (88/110) of the BL Lacs initially surveyed by HST have known redshifts, of which all 39 with $z<0.25$ and 21 of the 28 with $0.25<z<0.6$ have their hosts resolved, suggesting that the redshift of PG 1553+113 is indeed large. Recently, using a re-analysis of the HST snapshot survey of BL Lacs, Sbarufatti et al. (2005) claimed the dispersion of the absolute magnitude of BLLac hosts is sufficiently small that the measurement of the host-galaxy brightness allows a reliable estimate of their redshift. With the assumption that there is no strong evolution, these authors set a possible lower limit of $z>0.78$ for PG 1553+113. However, adding new STIS-HST data of $z>0.6$ BL Lacs to the snapshot survey, O'Dowd \& Urry (2005) found on the contrary that host galaxies of BL Lacs evolve strongly, making any photometric redshift determination questionable. A conservative upper limit $(z<0.74)$ was determined (Aharonian et al. 2006a) from the photon spectrum measured by HESS. The same limit was similarly determined from the MAGIC spectral measurement (Albert et al. 2007). Using both the MAGIC and HESS data, a stronger upper limit of $z<0.42$ was later reported (Mazin \& Goebel 2007) based on the assumption that there is no break in the intrinsic VHE spectrum of the object. The range of allowed redshift is clearly large enough that the significant effects of EBL absorption cannot be reliably removed from the observed VHE spectrum of PG $1553+113$. This correspondingly makes modeling the high-energy portion of its SED unreliable. A clear detection of the object's redshift would dramatically improve the understanding of PG $1553+113$. Further, if it were found to be distant, its VHE spectrum could potentially provide strong constraints on the poorly-measured EBL (assuming a reasonable intrinsic spectrum) and contribute to establishing the VHE $\gamma$-ray horizon.

Results from $17.2 \mathrm{~h}$ of new HESS observations of PG 1553+113 in 2006 are reported here. In addition, a reanalysis of the previously published 2005 HESS data $(7.6 \mathrm{~h})$, with an improved calibration of the absolute energy scale of the detector, is presented. HESS and the Suzaku X-ray satellite observed the blazar simultaneously in July 2006. The HESS results from this epoch are also discussed. This will enable, for the first time, future modeling of an SED determined from simultaneous observations at VHE and lower energies. Finally, results of

${ }^{1}$ Falomo \& Treves (1990) demonstrate the catalog redshift of $z=$ 0.36 is incorrect. a March 2006 VLT SINFONI spectroscopy campaign to determine the redshift of PG $1553+113$ are also reported.

\section{HESS observations and analysis technique}

A total of $30.3 \mathrm{~h}$ of HESS observations were taken, typically in 28 -min data segments (runs), on PG 1553+113 in 2005 and 2006. The pointing of the HESS telescopes was offset $\pm 0.5^{\circ}$ from the position of PG $1553+113$ during the observations. The useful exposure is $24.8 \mathrm{~h}$ live time, as 9 of the 66 runs fail the HESS data-quality selection criteria. The data are processed using the standard HESS calibration (Aharonian et al. 2004) and analysis tools (Benbow 2005). Soft cuts (Aharonian et al. $2006 a)^{2}$ are applied to select candidate $\gamma$-ray events. The soft cuts are selected instead of the standard cuts since they result in a lower energy threshold. Hence they are more appropriate given the very soft VHE spectra previously measured by both HESS ( $\Gamma=4.0$; Aharonian et al. 2006a) and MAGIC $(\Gamma=4.2$; Albert et al. 2007). At the mean zenith angle of the observations, $37^{\circ}$, the soft cuts result in an average post-analysis energy threshold $^{3}$ of $300 \mathrm{GeV}$. Events falling within a circular region of radius $\theta_{\text {cut }}=0.14^{\circ}$ centered on PG $1553+113$ are considered on-source data. The background (off-source data) is estimated simultaneously to the on-source data from events recorded within the same field of view (FoV) using the Reflected-Region method (Berge et al. 2007). Equation (17) from Li \& Ma (1983) is used to calculate the significance of any excess. The energy of each event is corrected (Aharonian et al. 2006b) using efficiencies determined from simulated and observed muons. This correction accounts for long-term variations in the absolute energy scale due to a changing optical throughput of the HESS system. The HESS results have been verified using independent calibration and analysis chains.

\section{HESS results}

The results of the HESS observations for each dark period in 2005 and 2006, as well as relevant totals for the observations, are given in Table 1 . The table shows the dark periods in which PG 1553+113 was observed, the MJD of the first and last night of observations, the dead time corrected observation time, the number of on- and off-source events, the on/off normalization $(\alpha)$, the measured excess, and the significance of the excess. A significant excess of events from the direction of PG $1553+113$ is clearly detected in each year of HESS data taking. A total of 785 excess events, corresponding to a statistical significance of 10.2 standard deviations $(\sigma)$, is detected in the complete data set. Figure 1 shows the on-source and normalized off-source distributions of the square of the angular difference between the reconstructed shower position and the source position $\left(\theta^{2}\right)$ for all observations. There is a clear point-like excess of on-source events at small values of $\theta^{2}$, corresponding to the observed signal, and the background is approximately flat in $\theta^{2}$ as expected. The peak of a two-dimensional Gaussian fit to a sky map of the observed excess is located at $\alpha_{\mathrm{J} 2000}=$ $15^{\mathrm{h}} 55^{\mathrm{m}} 44.7^{\mathrm{s}} \pm 3.0_{\text {stat }}^{\mathrm{s}} \pm 1.3_{\text {syst }}^{\mathrm{s}}, \delta_{\mathrm{J} 2000}=11^{\circ} 11^{\prime} 41^{\prime \prime} \pm 53_{\text {stat }}^{\prime \prime} \pm 20_{\text {syst }}^{\prime \prime}$ The excess, HESS J1555+111, is coincident with the position $\left(\alpha_{\mathrm{J} 2000}=15^{\mathrm{h}} 55^{\mathrm{m}} 43.0^{\mathrm{s}}, \delta_{\mathrm{J} 2000}=11^{\circ} 11^{\prime} 24.4^{\prime \prime}\right)$ of PG $1553+113$

\footnotetext{
2 The soft cuts were initially named the spectrum cuts in Aharonian et al. (2006a).

3 The threshold has been corrected to account for the decreased optical efficiency of the HESS mirrors.
} 
Table 1. Results of the HESS observations of PG $1553+113$.

\begin{tabular}{|c|c|c|c|c|c|c|c|c|c|c|c|c|}
\hline $\begin{array}{c}\text { Dark } \\
\text { Period }\end{array}$ & $\begin{array}{l}\text { MJD } \\
\text { First }\end{array}$ & $\begin{array}{l}\text { MJD } \\
\text { Last }\end{array}$ & $\begin{array}{c}\text { Time } \\
{[\mathrm{h}]}\end{array}$ & On & Off & $\alpha$ & Excess & $\begin{array}{l}\text { Sig } \\
{[\sigma]}\end{array}$ & $\begin{array}{c}I(>300 \mathrm{GeV})^{a} \\
{\left[10^{-12} \mathrm{~cm}^{-2} \mathrm{~s}^{-1}\right]}\end{array}$ & $\begin{array}{c}\mathrm{Crab}^{b} \\
\%\end{array}$ & $\chi^{2}, \mathrm{NDF}^{c}$ & $P\left(\chi^{2}\right)$ \\
\hline $04 / 2005$ & 53492 & 53507 & 4.9 & 1210 & 8154 & 0.125 & 191 & 5.5 & $4.78 \pm 1.18$ & 3.5 & $2.7,4$ & 0.61 \\
\hline $08 / 2005$ & 53609 & 53614 & 2.7 & 491 & 3462 & 0.125 & 58 & 2.5 & $10.19 \pm 5.46$ & 7.5 & $4.9,2$ & 0.09 \\
\hline $04 / 2006$ & 53849 & 53860 & 7.1 & 1811 & 12742 & 0.125 & 218 & 5.0 & $4.08 \pm 1.10$ & 3.0 & $5.3,8$ & 0.72 \\
\hline $07 / 2006$ & 53938 & 53943 & 10.1 & 2236 & 15341 & 0.125 & 318 & 6.7 & $4.33 \pm 0.94$ & 3.2 & $3.1,5$ & 0.68 \\
\hline 2005 & 53492 & 53614 & 7.6 & 1701 & 11616 & 0.125 & 249 & 6.0 & $5.44 \pm 1.23$ & 4.0 & $0.9,1$ & 0.33 \\
\hline 2006 & 53849 & 53943 & 17.2 & 4047 & 28083 & 0.125 & 536 & 8.3 & $4.22 \pm 0.72$ & 3.1 & $0.03,1$ & 0.86 \\
\hline Total & 53492 & 53943 & 24.8 & 5748 & 39699 & 0.125 & 785 & 10.2 & $4.56 \pm 0.62$ & 3.4 & $0.7,1$ & 0.39 \\
\hline
\end{tabular}

${ }^{a}$ The systematic error on the observed integral flux above $300 \mathrm{GeV}$ is $20 \%$ and is not shown.

${ }^{b}$ The integral flux percentage is calculated relative to the Crab Nebula flux above $300 \mathrm{GeV}$.

${ }^{c}$ The $\chi^{2}$, degrees of freedom (NDF), and $\chi^{2}$ probability $P\left(\chi^{2}\right)$ are given for a fit of a constant to $I(>300 \mathrm{GeV})$ binned nightly within a dark period, or monthly within a year, or yearly within the total.

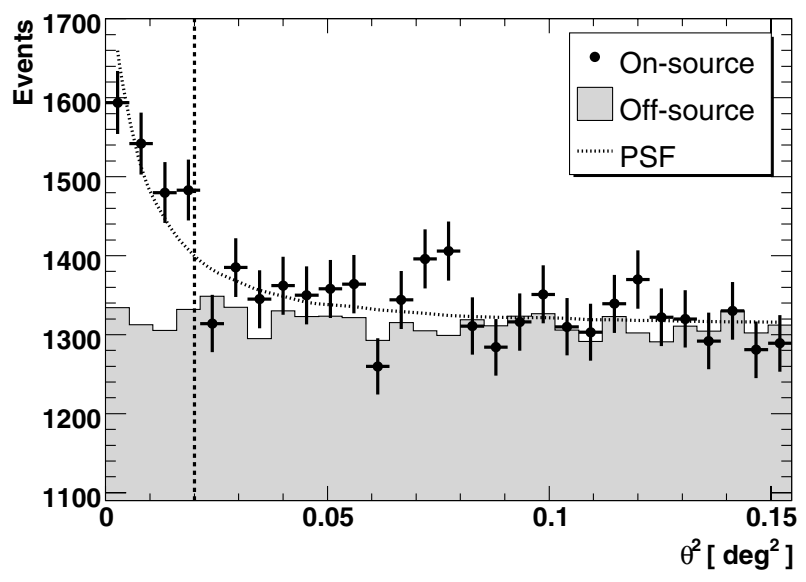

Fig. 1. Distribution of $\theta^{2}$ for on-source events (points) and normalized off-source events (shaded) from observations of PG 1553+113. The dashed curve represents the $\theta^{2}$ distribution expected for a point source of VHE $\gamma$-rays at $40^{\circ}$ zenith angle with a photon index $\Gamma=4.46$. The vertical line represents the cut on $\theta^{2}$ applied to the data.

(Beasley et al. 2002). The upper limit (99\% confidence level) on the extension of HESS J1555+111 is $1.1^{\prime}$.

\subsection{Spectral studies}

The photon spectrum for the entire data set is shown in Fig. 2. These data can be fit ( $\chi^{2}$ of 8.4 for 5 degrees of freedom) by a power-law function $\left.\mathrm{d} N / \mathrm{d} E=I_{\circ}(E / 300 \mathrm{GeV})^{-\Gamma}\right)$ with a photon index $\Gamma=4.46 \pm 0.34_{\text {stat }} \pm 0.10_{\text {syst }}$. Fits of either a power law with an exponential cut-off or a broken power law do not yield significantly better $\chi^{2}$ values. It should be noted that each of the three highest energy points, $E>0.6 \mathrm{TeV}$, in Fig. 2 have statistical significance less than $2 \sigma$. However, removing these points from the fit does not change $\Gamma$ significantly.

The significant photon statistics from each year of observations allows the determination of annual spectra. These spectra are shown in Fig. 3. The results of fits to the data of a power law for both years, as well as for the total, are shown in Table 2. The lower and upper energy bounds, photon index $(\Gamma)$, differential flux normalization at $300 \mathrm{GeV}\left(I_{\circ}\right), \chi^{2}$, degrees of freedom (NDF), and $\chi^{2}$ probability $P\left(\chi^{2}\right)$ for each fit are given in the table. There are no significant changes in the spectral shape during the HESS observations. By including one negative point at $\sim 600 \mathrm{GeV}$, and two more positive points at higher energies, the 2006 spectrum can be fit over the same range as either the 2005 or the overall spectrum. This fit yields $\Gamma \approx 4.9$,

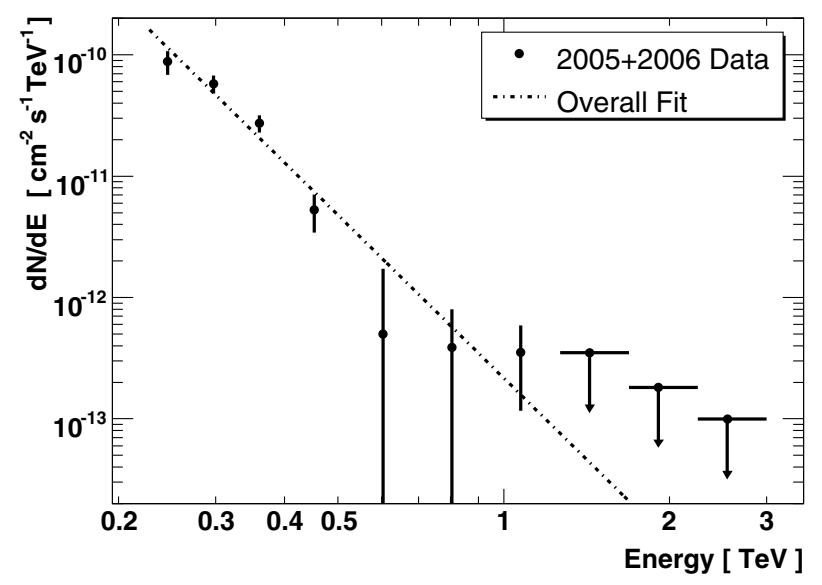

Fig. 2. Overall VHE energy spectrum observed from PG 1553+113. The dashed line represents the best $\chi^{2}$ fit of a power law to the observed data. The upper limits are at the $99 \%$ confidence level (Feldman \& Cousins 1998). Only the statistical errors are shown.

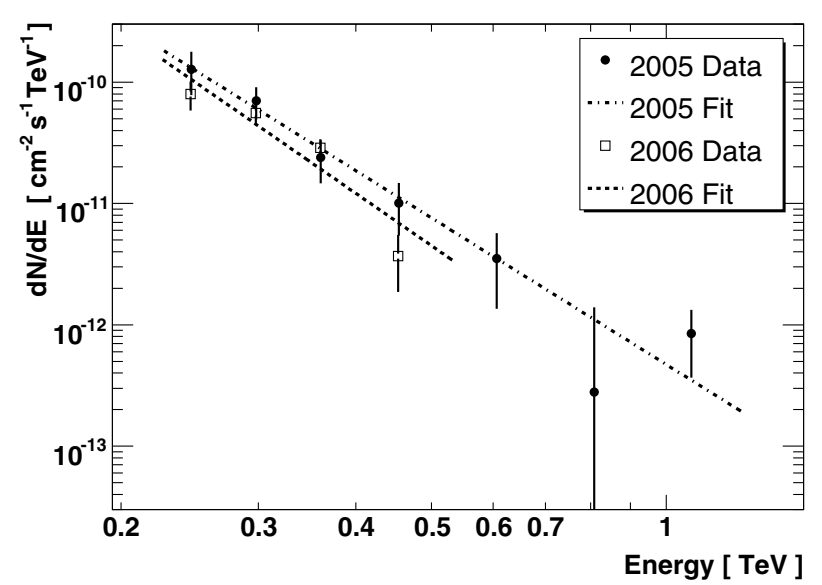

Fig. 3. Annual VHE energy spectra observed by HESS from PG $1553+113$. Each line represents the best $\chi^{2}$ fit of a power law to the observed data. Only the statistical errors are shown.

explaining why the overall photon index is slightly softer than the 2005 and 2006 indices reported in Table 2.

\subsection{Integral flux studies}

All HESS fluxes (i.e. annual, dark period and nightly values) throughout this article are calculated assuming the measured 
Table 2. Results of the best $\chi^{2}$ fit to the annual and overall spectra of PG $1553+113$ measured by HESS.

\begin{tabular}{cccccccc}
\hline \hline Epoch & $\begin{array}{c}E_{\min } \\
{[\mathrm{TeV}]}\end{array}$ & $\begin{array}{c}E_{\max } \\
{[\mathrm{TeV}]}\end{array}$ & $\Gamma$ & $\begin{array}{c}I_{\circ} \\
{\left[10^{-11} \mathrm{~cm}^{-2} \mathrm{~s}^{-1} \mathrm{TeV}^{-1}\right]}\end{array}$ & $\chi^{2}$ & $\mathrm{NDF}$ & $P\left(\chi^{2}\right)$ \\
\hline $2005(\mathrm{AH06})^{a}$ & 0.185 & 0.585 & $3.98 \pm 0.63_{\text {stat }} \pm 0.10_{\text {syst }}$ & $2.59 \pm 0.47_{\text {stat }} \pm 0.52_{\text {syst }}$ & 1.7 & 2 & 0.42 \\
\hline $2005^{b}$ & 0.225 & 1.265 & $4.01 \pm 0.60_{\text {stat }} \pm 0.10_{\text {syst }}$ & $5.92 \pm 1.19_{\text {stat }} \pm 1.18_{\text {syst }}$ & 2.1 & 5 & 0.84 \\
2006 & 0.225 & 0.534 & $4.45 \pm 0.48_{\text {stat }} \pm 0.10_{\text {syst }}$ & $4.35 \pm 0.54_{\text {stat }} \pm 0.87_{\text {syst }}$ & 8.5 & 2 & 0.014 \\
\hline Total & 0.225 & 1.265 & $4.46 \pm 0.34_{\text {stat }} \pm 0.10_{\text {syst }}$ & $4.68 \pm 0.49_{\text {stat }} \pm 0.94_{\text {syst }}$ & 8.4 & 5 & 0.13 \\
\hline
\end{tabular}

${ }^{a}$ The AH06 entry is the previously published HESS result (Aharonian et al. 2006a) for 2005. The AH06 entry was not corrected for long-term changes in the optical efficiency of the system.

${ }^{b}$ The 2005 entry corresponds to exactly the same data as presented in AH06, but with a correction (see text) applied to account for optical efficiency changes within the data. The 2006 and total entries also have this correction applied.

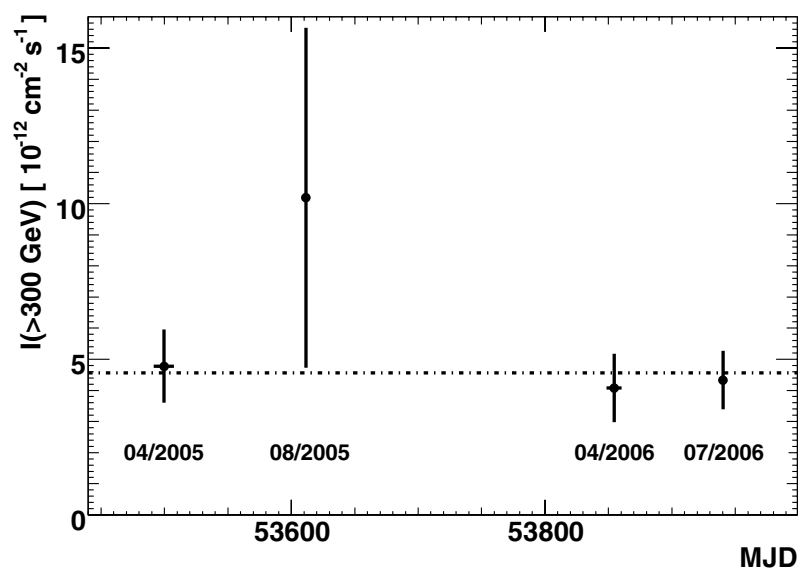

Fig. 4. Integral flux, $I(>300 \mathrm{GeV})$, measured by HESS from PG 1553+113 during each dark period of observations. The horizontal line represents the average flux for all the HESS observations. For each point the time-averaged $\Gamma=4.46$ is assumed. Only the statistical errors are shown.

time-average photon index of $\Gamma=4.46$. Assuming a different value (i.e. $4.0<\Gamma<5.0$ ) has less than a $5 \%$ effect on the flux. The observed integral flux above $300 \mathrm{GeV}$ for the entire data set is $I(>300 \mathrm{GeV})=\left(4.56 \pm 0.62_{\text {stat }} \pm 0.91_{\text {syst }}\right) \times 10^{-12} \mathrm{~cm}^{-2} \mathrm{~s}^{-1}$. This corresponds to $\sim 3.4 \%$ of $I(>300 \mathrm{GeV})$ determined from the HESS Crab Nebula spectrum (Aharonian et al. 2006b). The integral flux, $I(>300 \mathrm{GeV})$, is shown in Table 1 for each year of observations, as well as for each dark period. The $\chi^{2}$ and corresponding probability shown in the table is for a fit of a constant to the data when binned by nights within each dark period, by dark periods within a year, and by year within the total observations. There are no indications for flux variability on any time scale within the HESS data. Figures 4 and 5 show the flux measured for each dark period and night, respectively.

\subsection{Effect of the optical efficiency correction}

The data previously published (Aharonian et al. 2006a) for HESS observations of PG 1553+113 in 2005 were not corrected for long-term changes in the optical sensitivity of the instrument. Relative to a virgin telescope, the total optical throughput was decreased by $29 \%$ in 2005 and $33 \%$ in 2006 . These losses are due to the reduced reflectivity of both the mirrors and Winston cones, and to the accumulation of dust on the optical elements. Comparing (see Table 2) the spectrum for 2005 determined here to that which was previously published for the same data shows significant differences in the flux normalization $\left(I_{\circ}\right)$ but not in the photon index $(\Gamma)$. Figure 6 illustrates this difference which is a result of correcting the energy of individual events for the relative optical efficiency of the system, determined from simulated and observed muon events, as described earlier. The flux measured in 2005 is therefore three times higher than previously published ${ }^{4}$. The large difference is due to the steep spectrum of the source. For harder spectrum sources the effect is much smaller.

\subsection{Comparison to MAGIC results}

Table 3 shows the photon index and integral flux for PG 1553+113 measured by both the MAGIC (Albert et al. 2007) and HESS collaborations. As the data were not simultaneously measured, one might not expect the measured quantities to agree due to variability arguments. In addition, the spectra from the two instruments are measured over different energy ranges which could result in different spectral slopes if the spectrum were curved. Nevertheless, the spectral slope $(\Gamma)$ measured by both instruments agree within errors in all epochs. Further, the flux above $200 \mathrm{GeV}$ measured by both HESS and MAGIC in 2005 is consistent ${ }^{5}$. However the flux observed in 2006 by MAGIC is lower than the HESS value. It should be noted that in 2006, all the HESS data were taken after the final published MAGIC observations, whereas a good fraction of the 2005 data from both instruments are quasi-simultaneous.

\subsection{HESS results during Suzaku observations}

The Suzaku X-ray satellite (Mitsuda et al. 2007) observed PG 1553+113 from July 24, 2006, 14:26 UTC to July 25, 2006, 19:17 UTC with an average efficiency of $~ 53 \%$ (Suzaku Observation Log: http: //www . astro. isas .ac .jp/ suzaku/index.html.en). HESS observed PG 1553+113 for $3.1 \mathrm{~h}$ live time on the two dates of Suzaku observations. The HESS observation $\log$ on these dates, henceforth called the Suzaku epoch, is shown in Table 4. The log contains the start and stop times of the seven HESS observation runs from the two dates of Suzaku observations. In addition, the live time, observed excess and corresponding significance for each run are shown. All runs, except the last, are simultaneous with Suzaku

\footnotetext{
${ }^{4}$ The flux was reported above a threshold of $200 \mathrm{GeV}$. Extrapolating the earlier result, using $\Gamma=4.0$ as reported, yields $I(>300 \mathrm{GeV})=$ $\left(1.78 \pm 0.45_{\text {stat }} \pm 0.35_{\text {syst }}\right) \times 10^{-12} \mathrm{~cm}^{-2} \mathrm{~s}^{-1}$.

5 Previously the 2005 HESS result was $\sim 4$ times lower than the 2005 MAGIC result. The agreement is solely the result of the optical efficiency correction.
} 


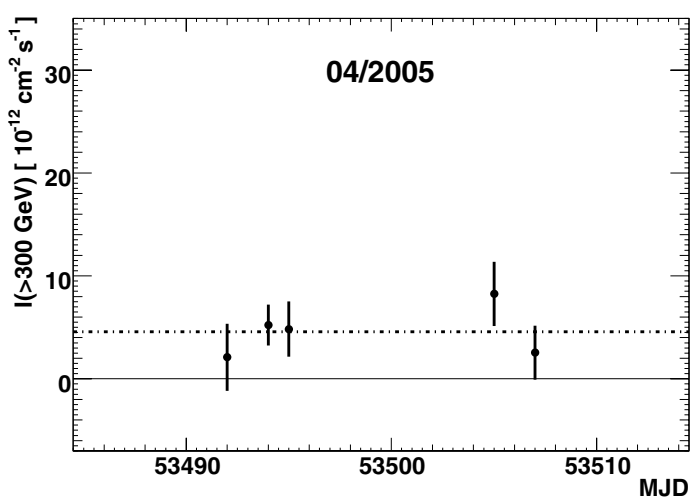

(a)

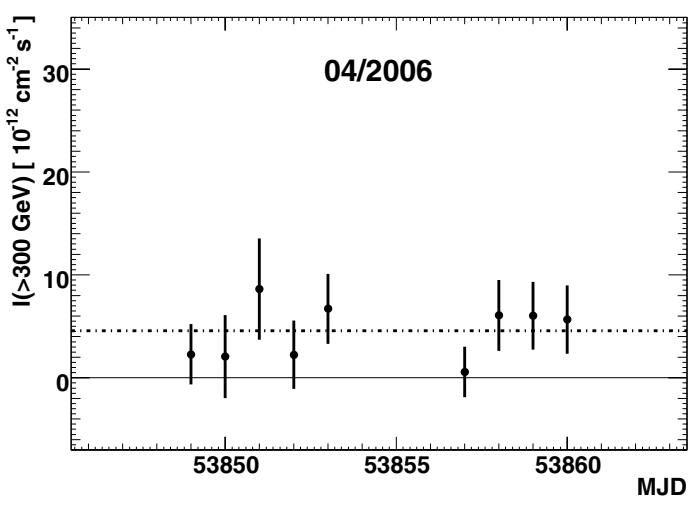

(c)

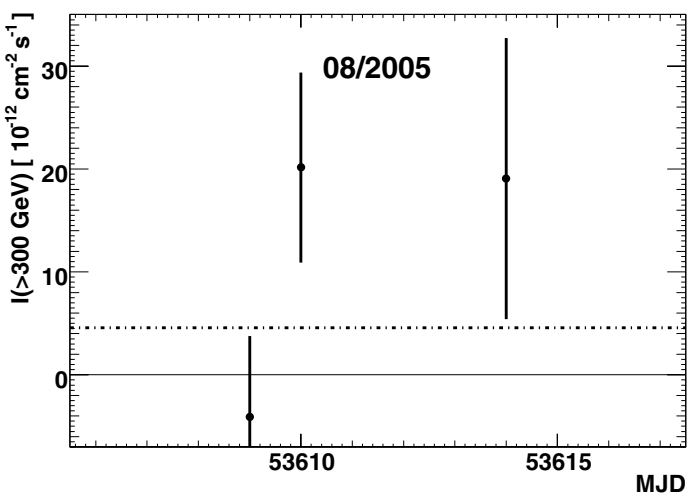

(b)

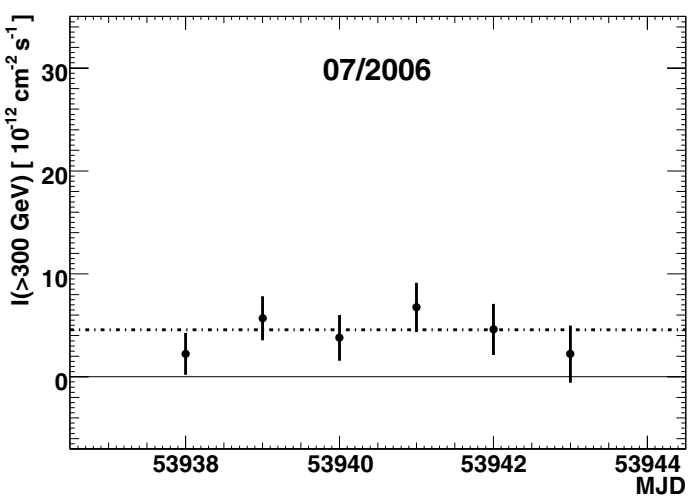

(d)

Fig. 5. Integral flux, $I(>300 \mathrm{GeV})$, measured by HESS from PG $1553+113$ during each night of observations. The plots a)-d), represent the April 2005, August 2005, April 2006 and July 2006 dark periods, respectively. The horizontal line represents the average flux for all the HESS observations. For each point the time-average $\Gamma=4.46$ is assumed. Only the statistical errors are shown.

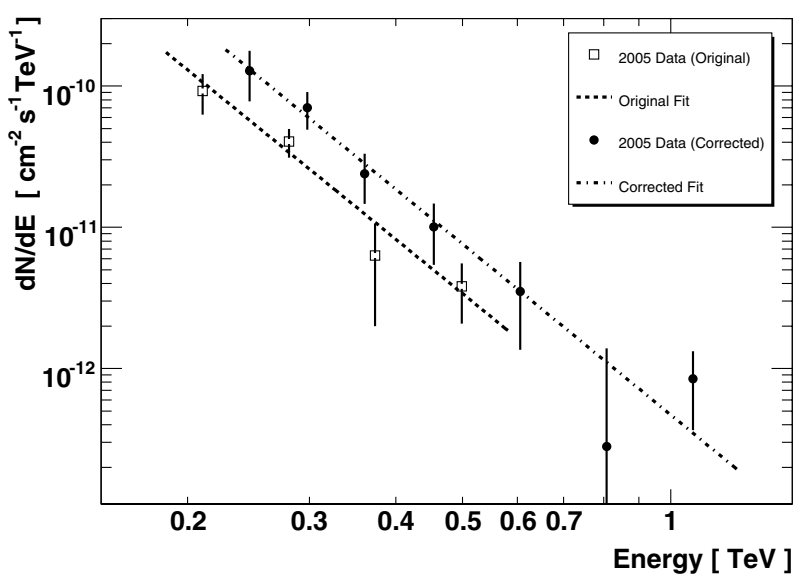

Fig. 6. Spectrum measured by HESS from PG $1553+113$ in 2005 compared to the previously published version (Aharonian et al. 2006a). The integral flux above $300 \mathrm{GeV}$ is three times higher. Only the statistical errors are shown.

observations. The last HESS run begins two minutes after the end of the Suzaku pointing.

During the Suzaku epoch, a total of 659 on-source events and 4462 off-source events are measured with an on-off normalization of 0.125 , corresponding to an excess of 101 events $(3.9 \sigma)$. Due to the low statistics it is not possible to produce a $\gamma$-ray spectrum from the data. To provide a quasi-simultaneous measurement for future modeling, the $\gamma$-ray spectrum observed during the July 2006 dark period is shown in Fig. 7a. The best $\chi^{2}$ fit of a power law to the July 2006 data yields $\Gamma=$ $5.0 \pm 0.7, I_{\circ}=3.6 \pm 0.6 \times 10^{-13} \mathrm{~cm}^{-2} \mathrm{~s}^{-1} \mathrm{TeV}^{-1}$, and a $\chi^{2}$ of 4.8 for 2 degrees of freedom. Only the statistical errors are presented for $\Gamma$ and $I_{\circ}$. The systematic errors are 0.1 and $20 \%$, respectively.

The average flux ${ }^{6}$ during the Suzaku epoch is $I(>300 \mathrm{GeV})=\left(5.8 \pm 1.7_{\text {stat }} \pm 1.2_{\text {syst }}\right) \times 10^{-12} \mathrm{~cm}^{-2} \mathrm{~s}^{-1}$. The flux for each of the nights in the Suzaku epoch can be seen in Fig. 5d. In addition, the flux for each HESS run ( $28 \mathrm{~min})$ during the two nights is shown in Fig. 7b. There are no significant variations of the run-wise flux during each night, or of the nightly flux during the Suzaku epoch.

\section{SINFONI near-IR spectroscopy}

The determination of the redshift of an AGN is generally based upon the detection of emission or absorption lines in its spectrum. In the unified model of AGN (Urry \& Padovani 1995) emission lines are generated by ionization from the central source. However in BL Lac objects like PG 1553+113 the lineequivalent width of these emission lines is reduced, possibly even below detectable levels, by the strong beaming of the jet continuum, that is caused by the alignment of the relativistic jet with the line of sight to Earth. Absorption lines can be produced

\footnotetext{
6 Assuming the poorly-measured $\Gamma=5.01$ from July 2006, instead of the time-averaged $\Gamma=4.46$, increases the flux by only $\sim 3.5 \%$.
} 
Table 3. Photon indices and integral fluxes measured by HESS and MAGIC.

\begin{tabular}{ccccc}
\hline \hline Epoch & $\begin{array}{c}\text { HESS }^{a} \\
\Gamma\end{array}$ & $\begin{array}{c}\text { HESS }^{b} \\
(>200 \mathrm{GeV}) \\
{\left[10^{-11} \mathrm{~cm}^{-2} \mathrm{~s}^{-1}\right]}\end{array}$ & $\begin{array}{c}\text { MAGIC } \\
\Gamma\end{array}$ & $\begin{array}{c}\text { MAGIC } \\
I(>200 \mathrm{GeV}) \\
{\left[10^{-11} \mathrm{~cm}^{-2} \mathrm{~s}^{-1}\right]}\end{array}$ \\
\hline 2005 & $4.01 \pm 0.60_{\text {stat }}$ & $2.21 \pm 0.50_{\text {stat }}$ & $4.31 \pm 0.45_{\text {stat }}$ & $2.0 \pm 0.6_{\text {stat }}$ \\
2006 & $4.45 \pm 0.48_{\text {stat }}$ & $1.72 \pm 0.29_{\text {stat }}$ & $3.95 \pm 0.35_{\text {stat }}$ & $0.6 \pm 0.2_{\text {stat }}$ \\
\hline $2005+2006$ & $4.46 \pm 0.34_{\text {stat }}$ & $1.85 \pm 0.25_{\text {stat }}$ & $4.21 \pm 0.25_{\text {stat }}$ & $1.0 \pm 0.4_{\text {stat }}$ \\
\hline
\end{tabular}

${ }^{a}$ The systematic error on the HESS $\Gamma$ is 0.1 .

${ }^{b}$ The HESS fluxes are extrapolated from the values in Table 1 to above $200 \mathrm{GeV}$ using the time-average $\Gamma=4.46$. The systematic error on the HESS flux is $20 \%$.

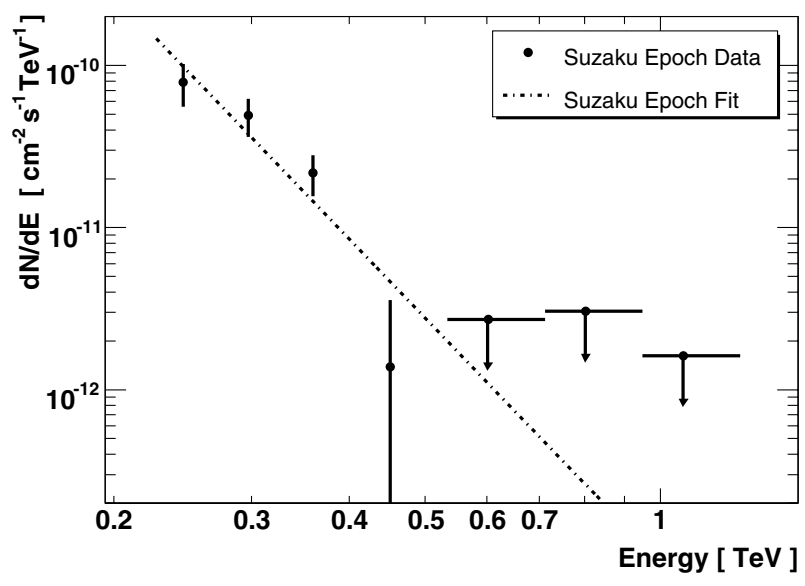

(a)

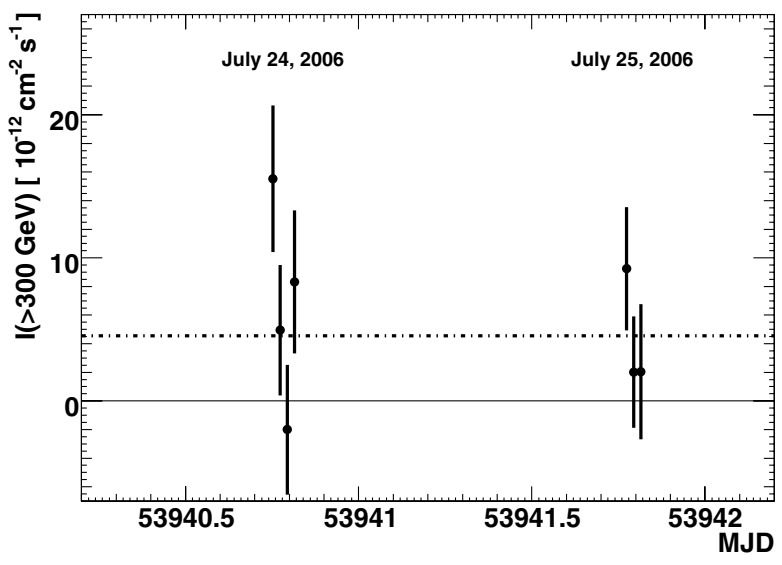

(b)

Fig. 7. a) Spectrum observed by HESS from PG $1553+113$ during observations in July 2006. The dashed line represents the best $\chi^{2}$ fit of a power law to the observed data. The upper limits are at the $99 \%$ confidence level (Feldman \& Cousins 1998). b) Integral flux, $I(>300 \mathrm{GeV}$ ), measured by HESS from PG 1553+113 in each run taken during simultaneous Suzaku observations. The horizontal line represents the average flux for all the HESS observations. For each point in the light curve the time-average $\Gamma=4.46$ is assumed. For both plots, only the statistical errors are shown.

Table 4. HESS observation log during the Suzkau epoch.

\begin{tabular}{ccccc}
\hline \hline $\begin{array}{c}\text { Start } \\
{[\mathrm{MJD}]}\end{array}$ & $\begin{array}{c}\text { Stop } \\
{[\mathrm{MJD}]}\end{array}$ & $\begin{array}{c}\text { Time } \\
{[\mathrm{h}]}\end{array}$ & Excess & $\begin{array}{c}\text { Significance } \\
{[\sigma]}\end{array}$ \\
\hline 53940.74231 & 53940.76185 & 0.453 & 27 & 2.5 \\
53940.76328 & 53940.78277 & 0.436 & 20 & 1.9 \\
53940.78431 & 53940.80385 & 0.437 & 7 & 0.7 \\
53940.80531 & 53940.82480 & 0.435 & -2 & -0.2 \\
53941.76336 & 53941.78291 & 0.444 & 27 & 3.1 \\
53941.78439 & 53941.80392 & 0.444 & 14 & 1.6 \\
53941.80542 & 53941.82497 & 0.443 & 7 & 0.8 \\
\hline
\end{tabular}

either from spectral features of the stellar population of the host galaxy or from intervening halos as in the case of quasars. The detectability of stellar spectral features depends inversely on the brightness of the central source. Therefore, the probability of detection of any spectral features is likely optimal in the near infrared (IR) wavelength range. Here, the stellar contribution to the overall continuum emission is much larger than at optical or mid-IR wavelengths due to the shape of the SEDs of the AGN and the stars. In addition, the extinction affecting the stellar light is much smaller at near-IR wavelengths than at optical (e.g. $A_{H} \sim 0.17 A_{V}$ ) wavelengths (Schlegel et al. 1998).

In an attempt to detect absorption features from the host galaxy or emission lines from the AGN, $H+K$ $(1.50-2.40 \mu \mathrm{m})$ spectroscopy of PG $1553+113$ was performed with SINFONI, an integral field spectrometer mounted at Yepun,
Unit Telescope 4 of the ESO Very Large Telescope in Chile. The observations were performed in seeing-limited mode using the 125 mas pixel scale, with a spectral resolution of $R_{H+K} \sim$ 1500 . The seeing was typically 0 !' 7 FWHM during the 2.5 total hours of on-source observations performed on March 9, 2006 and March 15, 2006.

The ESO Data Reduction Pipeline Version 1.3 (Jung et al. 2006) and the QFitsView ${ }^{7}$ software are used for the data analysis. In the first steps, the images are cleaned from bad pixels, flat-fielded and wavelength calibrated. A distortion correction is then applied and the FoV of SINFONI is reconstructed. The output of this procedure is a three-dimensional (3D) cube containing about 2000 images of the source in $0.5 \mathrm{~nm}$ wavelength steps.

Averaging the cube along the wavelength axis enables the effective construction of a broad-band image. $H$-band and $K$-band images were thus extracted from PG $1553+113$ data. These images are spatially unresolved and no underlying host galaxy is detected.

A spectrum is extracted from the final 3D cube by summing the individual spectra within an aperture of 5-pixel radius centered on the brightest peak in the image. Observations of two G2V standard stars (HIP 071527, HIP 088235), near both in time and air-mass to the target exposures, are used to correct for strong atmospheric (telluric) absorption. By dividing the science target spectrum by the standard star spectrum, the

\footnotetext{
7 Written by Thomas Ott (MPE, Garching);
} http://www . mpe.mpg.de/ ott/QFitsView 


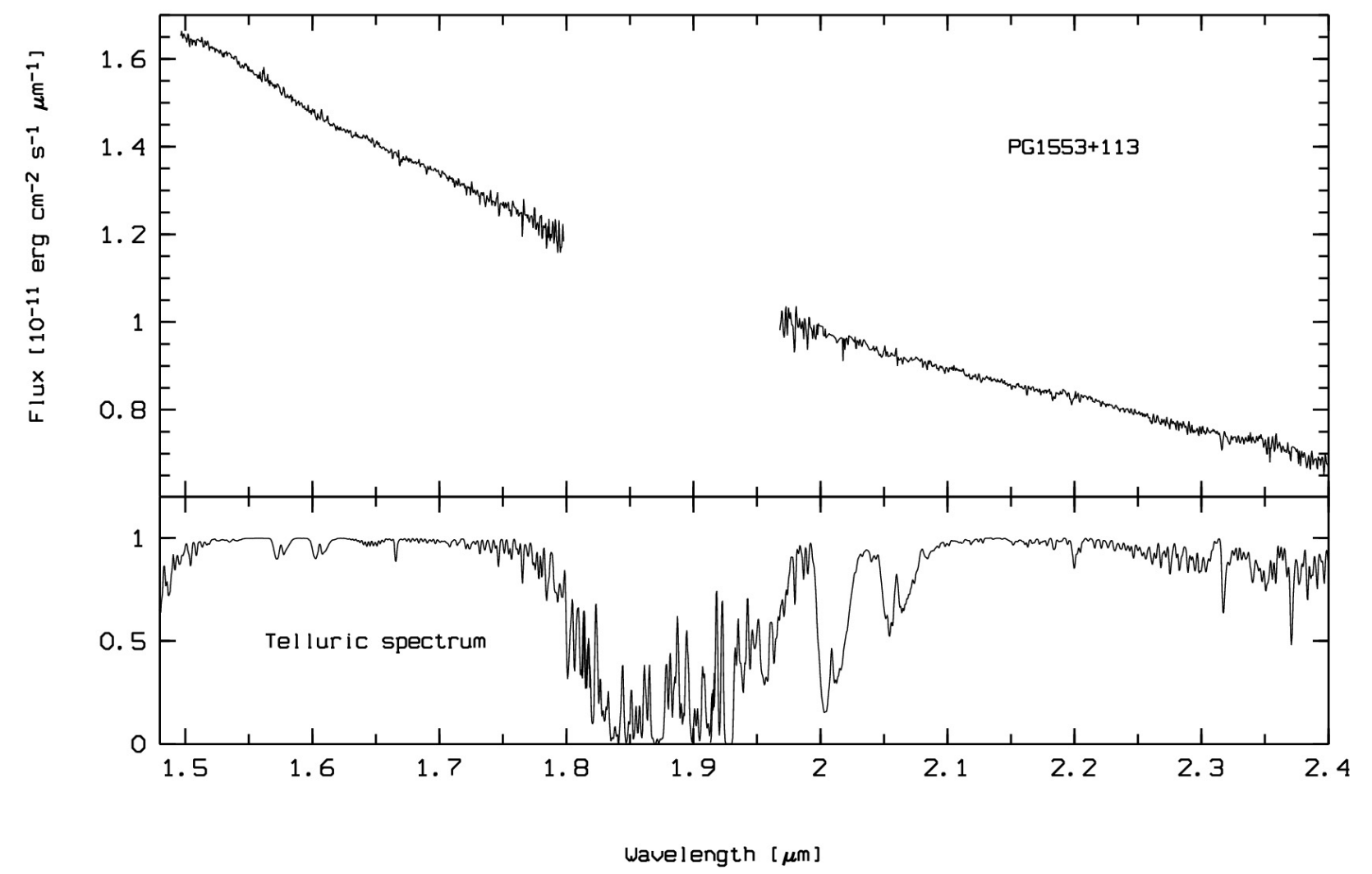

Fig. 8. Top: $H+K$-band spectrum of PG $1553+113$ extracted from a 5-pixel radius aperture. The gap is due to the highly reduced atmospheric transmission between $H$ and $K$ bands. Bottom: telluric spectrum extracted from the standard stars.

atmospheric absorption effects are minimized (see, e.g., Maiolino et al. 1996; Vacca et al. 2003). However, this step introduces features into the resulting spectrum that are intrinsic to the standard star. Here, these features are accounted for by multiplication of the normalized high-spectral-resolution atmospherictransmission-corrected solar spectrum ${ }^{8}$ that has been degraded to the SINFONI spectral resolution.

Flux calibration at infrared wavelengths is somewhat more problematic than at optical wavelengths since no standard spectral catalog exists. A theoretical continuum spectrum of a G2V star could in principle be used to correct for the throughput of the spectrograph. However, the flux distribution of such stars, as well as later-type ones, in the infrared is not well-reproduced by theoretical modeling, and a small error in the stellar parameters (e.g., the effective temperature or the metallicity) can yield a large error in the continuum. Instead the wavelength-dependent flux calibration is determined from observations of two intrinsically featureless (apart from strong hydrogen lines) B stars (HIP 088201, HIP 085812). Here the hydrogen lines are manually fit and removed from the B-star spectrum, and the result is then divided by a blackbody spectrum at the effective temperature of the stars $(18500 \mathrm{~K}$ and $11900 \mathrm{~K}$, respectively) to correct for the continuum slope of each standard star. As the $H$ and $K$ bands are in the Rayleigh-Jeans regime of the blackbody spectrum, the steepness of the continuum slope for these B stars is not particularly sensitive to small errors in the effective temperature. The final step of the flux calibration is setting the absolute flux scale. IR aperture photometry of PG $1553+113$

${ }^{8} \mathrm{NSO} /$ Kitt Peak FTS data of the sun used here were produced by NSF/NOAO. was already performed but the source is known to be variable. Therefore 2MASS H magnitudes of the standard stars, converted to flux density, are used instead to scale the total flux observed here. This value, $12.7 \pm 1.3 \mathrm{mJy}$ at $1.65 \mu \mathrm{m}$, is approximately half of that observed by Bersanelli et al. (1992) and is comparable with $R$-band fluxes observed (Albert et al. 2007) in March 2006. It should be noted that the latter (March 15, 2006) of the near-IR observations reported here were performed two days before an outburst in the $R$-band reported by Albert et al. (2007).

The final $H+K$-band spectrum of PG $1553+113$ is shown in Fig. 8. The signal-to-noise ratio is $\sim 250$ in the $H$-band and $\sim 70$ in the $K$-band. These measurements are the most sensitive ever recorded in the $H$ - and $K$-bands, and are comparable to the most sensitive spectroscopy of PG $1553+113$ at other wavelengths (see, e.g., Carangelo et al. 2003; Sbarufatti et al. 2006). The lack of data between $\sim 1.80-1.95 \mu \mathrm{m}$ is a result of highly-reduced atmospheric transmission between the $H$ and $K$ bands. The observed near-IR spectrum is featureless apart from some residuals from the atmospheric correction. Thus, in neither the broad-band images nor in the spectrum are the influences of the gas of a host galaxy or the AGN detected, even though PG 1553+113 is bright in the IR. As a result, a redshift determination from these observations is not possible.

\section{Discussion}

The presence of Suzaku X-ray observations, simultaneous with the HESS measurements, greatly improves the possibilities for accurately modeling the underlying physics in PG $1553+113$. However, the observed VHE spectrum is very soft $(\Gamma=4.46 \pm$ 0.34 ), which could be in large part due to the redshift-dependent 
absorption of VHE photons on the EBL. Modeling the VHE portion of the SED of PG $1553+113$ requires the effects of this absorption to be removed. Unfortunately the redshift of the object is still unknown, despite the very sensitive new measurements presented in this article. Therefore, modeling of the VHE emission from PG $1553+113$ is extremely difficult given the large range of possible intrinsic spectra.

PG $1553+113$ is not the only bright BL Lac for which the underlying host galaxy is not detected, even though deep, high signal-to-noise ratio observations were conducted (e.g., O'Dowd \& Urry 2005; Sbarufatti et al. 2006) for many. The properties of their host galaxy or their AGN may be such that it is presently not possible to derive their intrinsic nature and hence redshift. In these cases, it is possible that the properties of the host are not those of giant elliptical galaxies, but correspond to the faint end of the elliptical luminosity distribution $\left(M_{R} \sim-20\right)$ or that the AGN may even be hosted by a dwarf galaxy, making these BL Lac objects the radio-loud equivalent to the low-mass blackhole Seyfert galaxies (Barth et al. 2005). If this is the case, it will be very difficult to derive a firm redshift for PG $1553+113$.

As mentioned in the introduction, it is possible to derive upper limits on the object's redshift using VHE measurements, some basic assumptions about the source's intrinsic spectrum, and an EBL density model. As yet, none of the limits derived in this fashion are particularly constraining. The strongest, and also based on the most assumptions, is $z<0.42$ (Mazin \& Goebel 2007). Following exactly the same methodology as in Aharonian et al. (2006a), the VHE spectrum measured here from PG $1553+113$ limits the redshift to $z<0.69$.

\section{Conclusion}

With a data set that is $\sim 3$ times larger than previously published (Aharonian et al. 2006a), the HESS signal from PG 1553+113 is now highly significant $(\sim 10 \sigma)$. Thus, the evidence for VHE emission previously reported is clearly verified. However, the flux measured in 2005 is now $\sim 3$ times higher than first reported due to an improved calibration of the absolute energy scale of HESS. The statistical error on the VHE photon index is now reduced from $\sim 0.6$ to $\sim 0.3$. Nevertheless, the error of 0.34 is still rather large, primarily due to the extreme softness of the observed spectrum $(\Gamma=4.46)$. The total HESS exposure on PG $1553+113$ is $\sim 25 \mathrm{~h}$. Barring a flaring episode, not yet seen in two years of observations, a considerably larger total exposure $(\sim 100 \mathrm{~h})$ would be required to significantly improve the spectral measurement. This large exposure is unlikely to be quickly achieved. However, the VHE flux from other AGN is known to vary dramatically and even a factor of a few would reduce the observation requirement considerably. Should such a VHE flare occur, not only will the error on the measured VHE spectrum be smaller, but the measured photon index may also be harder (see, e.g., Aharonian et al. 2002). Both effects would dramatically improve the redshift constraints and correspondingly the accuracy of the source modeling. Therefore, the VHE flux from PG $1553+113$ will continue to be monitored by HESS. In addition, the soft VHE spectrum makes it an ideal target for the lower-threshold HESS Phase-II (Punch 2005) which should make its first observations in 2009.

Acknowledgements. The support of the Namibian authorities and of the University of Namibia in facilitating the construction and operation of HESS is gratefully acknowledged, as is the support by the German Ministry for Education and Research (BMBF), the Max Planck Society, the French Ministry for Research, the CNRS-IN2P3 and the Astroparticle Interdisciplinary Programme of the CNRS, the UK Science and Technology Facilities Council (STFC), the
IPNP of the Charles University, the Polish Ministry of Science and Higher Education, the South African Department of Science and Technology and National Research Foundation, and by the University of Namibia. We appreciate the excellent work of the technical support staff in Berlin, Durham, Hamburg, Heidelberg, Palaiseau, Paris, Saclay, and in Namibia in the construction and operation of the equipment. Based on ESO-VLT SINFONI program 276.B-5036 observations.

\section{References}

Albert, J., Aliu, E., Anderhub, H., et al. 2007, ApJ, 654, L119

Aharonian, F., Akhperjanian, A., Beilicke, M., et al. (HEGRA Collaboration) 2002, A\&A, 393, 89

Aharonian, F., Akhperjanian, A. G., Aye, K.-M., et al. (HESS Collaboration) 2004, Astropart. Phys., 22, 109

Aharonian, F., Akhperjanian, A. G., Bazer-Bachi, A. R., et al. (HESS Collaboration) 2006a, A\&A, 448, L19

Aharonian, F., Akhperjanian, A. G., Bazer-Bachi, A. R., et al. (HESS Collaboration) 2006b, A\&A, 457, 899

Barth, A. J., Greene, J. E., \& Ho, L. C. 2005, ApJ, 619, 151

Beasley, A. J., Gordon, D., Peck, A. B., et al. 2002, ApJS, 141, 13

Benbow, W. 2005, Proceedings of Towards a Network of Atmospheric Cherenkov Detectors VII (Palaiseau), 163

Berge, D., Funk, S., \& Hinton, J. 2007, A\&A, 466, 1219

Bersanelli, M., Bouchet, P., Falomo, R., \& Tanzi, E. G. 1992, AJ, 104, 28

Carangelo, N., Falomo, R., Kotilainen, J., Treves, A., \& Ulrich, M.-H. 2003, ASP Conf. Ser., 299, 299

Falomo, R., \& Treves, A. 1990, PASP, 102, 1120

Feldman, G. J., \& Cousins, R. D. 1998, Phys. Rev. D, 57, 3873

Giommi, P., Ansari, S. G., \& Micol, A. 1995, A\&AS, 109, 267

Gould, R. J., \& Schréder, G. P. 1967, Phys. Rev., 155, 1408

Hauser, M. G., \& Dwek, E. 2001, ARA\&A, 39, 249

Jung, Y., Lundin, L. K., Modigliani, A., Dobrzycka, D., \& Hummel, W. 2006, ASP Conf. Ser., 351, 295

Li, T., \& Ma, Y. 1983, ApJ, 272, 317

Maiolino, R., Rieke, G. H., \& Rieke, M. J. 1996, AJ, 111, 537

Mazin, D., \& Goebel, F. 2007, ApJ, 655, L13

Mitsuda, K., Bautz, M., Inoue, H., et al. 2007, PASJ, 59, 1

O'Dowd, M., \& Urry, C. M. 2005, ApJ, 627, 97

Osterman, M. A., Miller, H. A., Campbell, A. M., et al. 2006, AJ, 132, 873

Punch, M. 2005, Proceedings of Towards a Network of Atmospheric Cherenkov Detectors VII (Palaiseau), 509

Sbarufatti, B., Treves, A., \& Falomo, R. 2005, ApJ, 635, 173

Sbarufatti, B., Treves, A., Falomo, R., et al. 2006, AJ, 132, 1

Scarpa, R., Urry, C. M., Falomo, R., et al. 2000, ApJ, 532, 740

Schlegel, D. J., Finkbeiner, D. P., \& Davis, M. 1998, ApJ, 550, 525

Urry, C. M., \& Padovani, P. 1995, PASP, 107, 803

Vacca, W. D., Cushing, M. C., \& Rayner, J. T. 2003, PASP, 115389

1 Max-Planck-Institut für Kernphysik, PO Box 103980, 69029

Heidelberg, Germany

e-mail: Wystan. Benbow@mpi-hd.mpg.de

2 Yerevan Physics Institute, 2 Alikhanian Brothers St., 375036

Yerevan, Armenia

3 Centre d'Étude Spatiale des Rayonnements, CNRS/UPS, 9 Av. du

Colonel Roche, BP 4346, 31029 Toulouse Cedex 4, France

4 Universität Hamburg, Institut für Experimentalphysik, Luruper Chaussee 149, 22761 Hamburg, Germany

5 Institut für Physik, Humboldt-Universität zu Berlin, Newtonstr. 15, 12489 Berlin, Germany

6 LUTH, Observatoire de Paris, CNRS, Université Paris Diderot, 5 place Jules Janssen, 92190 Meudon, France

e-mail: Catherine.Boisson@obspm.fr

7 DAPNIA/DSM/CEA, CE Saclay, 91191 Gif-sur-Yvette Cedex, France

${ }^{8}$ University of Durham, Department of Physics, South Road, Durham DH1 3LE, UK

9 Unit for Space Physics, North-West University, Potchefstroom 2520, South Africa

10 Laboratoire Leprince-Ringuet, École Polytechnique, CNRS/IN2P3, 91128 Palaiseau, France

11 Laboratoire d'Annecy-le-Vieux de Physique des Particules, CNRS/IN2P3, 9 Chemin de Bellevue, BP 110, 74941 Annecy-leVieux Cedex, France 
12 APC, 11 place Marcelin Berthelot, 75231 Paris Cedex 05, UMR 7164; CNRS, Université Paris VII, CEA, Observatoire de Paris, France

${ }_{13}$ Dublin Institute for Advanced Studies, 5 Merrion Square, Dublin 2, Ireland

${ }^{14}$ Landessternwarte, Universität Heidelberg, Königstuhl, 69117 Heidelberg, Germany

15 Laboratoire de Physique Théorique et Astroparticules, CNRS/IN2P3, Université Montpellier II, CC 70, Place Eugène Bataillon, 34095 Montpellier Cedex 5, France

16 Universität Erlangen-Nürnberg, Physikalisches Institut, ErwinRommel-Str. 1, 91058 Erlangen, Germany

${ }_{17}$ Laboratoire d'Astrophysique de Grenoble, INSU/CNRS, Université Joseph Fourier, BP 53, 38041 Grenoble Cedex 9, France

${ }_{18}$ Institut für Astronomie und Astrophysik, Universität Tübingen, Sand 1, 72076 Tübingen, Germany
19 LPNHE, Université Pierre et Marie Curie Paris 6, Université Denis Diderot Paris 7, CNRS/IN2P3, 4 place Jussieu, 75252 Paris Cedex 5, France

20 Institute of Particle and Nuclear Physics, Charles University, V Holesovickach 2, 18000 Prague 8, Czech Republic

${ }^{21}$ Institut für Theoretische Physik, Lehrstuhl IV: Weltraum und Astrophysik, Ruhr-Universität Bochum, 44780 Bochum, Germany

22 University of Namibia, Private Bag 13301, Windhoek, Namibia

${ }^{23}$ Obserwatorium Astronomiczne, Uniwersytet Jagielloński, Kraków, Poland

24 Nicolaus Copernicus Astronomical Center, Warsaw, Poland

25 School of Physics \& Astronomy, University of Leeds, Leeds LS2 9JT, UK

${ }^{26}$ School of Chemistry \& Physics, University of Adelaide, Adelaide 5005, Australia

27 European Associated Laboratory for Gamma-Ray Astronomy, jointly supported by CNRS and MPG, Germany 